\title{
Accounting for PMD Temporal Correlation During Lightpath Set Up in Transparent Optical Networks
}

\author{
N. Sambo, M. Secondini, N. Andriolli, M. N. Petersen, and P. Castoldi, Member, IEEE
}

\begin{abstract}
In transparent optical networks, the signal transmission is degraded by optical layer physical impairments. Therefore, lightpaths may be blocked due to unacceptable quality of transmission (QoT). Among physical impairments, polarization mode dispersion (PMD) is a detrimental effect which has stochastic characteristics. Moreover, PMD depends on time-variant factors, such as the temperature and the fiber stress.

When implementing a dynamic GMPLS-controlled transparent optical network, the GMPLS protocol suite must take into account physical impairment information in order to establish lightpaths while guaranteeing the required QoT. In the literature, solutions for QoT-aware GMPLS control plane commonly consider that the effects of PMD on QoT are not detrimental when the average differential group delay (DGD) does not exceed a threshold. However, even with a high average DGD, it may happen that the instantaneous DGD is not detrimental. Additionally, given PMD temporal correlation properties, once that the instantaneous DGD is not detrimental, it continues to be not detrimental within considerable time ranges. Therefore, more accurate models can be implemented in the GMPLS control plane to account for PMD.

In this paper we propose a novel lightpath provisioning scheme based on a PMD prediction model which accounts for PMD temporal correlation properties. The proposed PMD-temporal-correlation (PTC) based lightpath provisioning scheme is compared with a scheme based on a classical PMD model. Simulation results show that PTC scheme significantly reduces the lightpath blocking probability with respect to the classical scheme. Moreover, PTC demonstrates that, by considering PMD temporal correlation, the transparency domain size can be increased, since paths that would be rejected by a classical model can be actually accepted within specific time ranges.
\end{abstract}

Index Terms-Control plane, GMPLS, physical impairments, PMD, transparent optical networks.

\section{INTRODUCTION}

$\mathbf{T}$ RANSPARENT optical networks are affected by optical layer physical impairments that cumulate along the paths. Therefore, some all-optical paths in the network may be unfeasible in terms of quality of transmission (QoT). In a dynamic transparent optical network, the QoT must be on-line evaluated upon lightpath request by means of estimation [1]-[6] or

Manuscript received February 22, 2010; revised September 08, 2010; accepted September 20, 2010. Date of publication September 27, 2010; date of current version November 17, 2010. This work was supported in part by the BONE-project ("Building the Future Optical Network in Europe"), a Network of Excellence funded by the European Commission through the 7th ICT-Framework Programme.

N. Sambo, M. Secondini, N. Andriolli, and P. Castoldi are with Scuola Superiore Sant'Anna, Pisa 56124, Italy (e-mail: nicola.sambo@sssup.it).

M. N. Petersen is with DTU Fotonik, Technical University of Denmark, Lyngby 2800 , Denmark.

Digital Object Identifier 10.1109/JLT.2010.2081344 measurements [7], [8]. To cope with this issue, physical impairments must be modeled, monitored, and considered during routing and wavelength assignment, and lightpath set up [2], [4]. Moreover, in case of distributed GMPLS-controlled networks, GMPLS routing protocol [2] or signaling protocol [4] must be extended to distribute physical impairment information needed to perform QoT estimation at each node. The solutions exploiting routing protocol (e.g., OSPF-TE) extensions require that QoT parameters related to links and node ports are flooded in the whole network and stored at each node. This represents a heavy burden to the control plane, although in a network only few paths (typically the longest ones) have to be avoided because of their unacceptable QoT. For this reason, the Internet Engineering Task Force (IETF) is discussing more scalable solutions based on the signaling protocol [9] (e.g., RSVP-TE). In this case each node needs to store QoT parameters related only to local ports and attached links, and QoT parameters, gathered along the computed path by the extended signaling protocol, allow to estimate the QoT during lightpath set up. Then, if the estimated QoT is acceptable, the lightpath is established, otherwise another set up attempt over a different path is performed. A further approach exploits the path computation element (PCE) architecture to perform the QoT estimation [10], with QoT parameters obtained from other sources (e.g., Network Management System) than the control plane. However, this scenario is outside the scope of the paper, which focuses on distributed schemes.

QoT in dynamic GMPLS-controlled transparent networks can be also evaluated through measurements on probe traffic [8]. In this way, the lightpath QoT is assessed during the lightpath establishment by sending probe traffic along the candidate path. Then, if the measured QoT on probe traffic is acceptable, the path is validated and client data traffic can be finally transmitted, otherwise another set up over a different path is attempted.

Considering QoT estimation, the authors in [3] identify amplified spontaneous emission (ASE), polarization mode dispersion (PMD), chromatic dispersion (CD), and self phase modulation (SPM) as the most relevant physical impairments to be modeled. Among them, PMD is a time-variant stochastic effect that depends on environmental (e.g., temperature, stress) and aging effects. Therefore, PMD may cause a channel outage along a path in specific time intervals, while it could be not so detrimental in other time intervals. In [3], a path is feasible in terms of PMD if the cumulated average differential group delay (DGD) $\bar{\tau}$ is below a maximum value, which ensures that the outage probability is acceptable. However, this condition can be pessimistic since, given a high $\bar{\tau}$, the instantaneous DGD may 
be not excessively detrimental in specific time intervals. Moreover, in [11] measurements on a $150 \mathrm{~km}$ span with $\bar{\tau}=0.64 \mathrm{ps}$ show that during quiescent periods, the DGD does not change more than 0.05 ps. Temporal dynamics of the PMD vector are also investigated in [12]-[14].

In this paper a QoT-aware lightpath provisioning scheme, based on both QoT estimation through RSVP-TE extensions and probe measurements, is proposed for GMPLS-controlled transparent optical networks. The novelty of the proposed scheme consists of improving QoT estimation by means of a PMD temporal correlation model. The adopted model, valid for any bit rate and WDM or single channel scenarios, aims at predicting PMD first-order dynamics and thus the lightpath outage probability in PMD-uncompensated systems. In particular, given the average path DGD and the measured DGD at time $t_{0}$, the model provides an estimation of the outage probability due to PMD within a time range $\left[t_{0} ; t_{0}+\Delta t\right]$. The proposed PMD-temporal-correlation (PTC) based lightpath provisioning scheme for WDM networks works as follows. First, the optical signal to noise ratio (OSNR) for the lightpath is estimated through RSVP-TE extensions. Then, the lightpath is set up, and probe traffic is sent along the lightpath for measuring the instantaneous DGD [15]-[17]. The measured DGD value is used by the proposed model, which accounts for PMD temporal evolutions, to assess the effects of PMD on QoT. The PTC scheme is compared with a classical RSVP-TE extensions based scheme [4] that utilizes an uncorrelated PMD model as in [3]. Simulation results show that PTC allows to significantly reduce the blocking probability. Moreover, PTC permits to establish lightpaths along paths which are rejected by the uncorrelated model, thus increasing the transparency domain size.

\section{PMD MODEL ACCOUNTING FOR PMD TEMPORAL CORRELATION}

We assume that a path is feasible in terms of PMD if, during the lightpath holding time, the outage probability - that we define as the probability that the instantaneous DGD $\tau(t)$, i.e., the modulus of the PMD vector $\vec{\tau}$, exceeds a maximum value $\tau_{\text {max }}$-is below a given threshold $P_{\text {th }}$. In a classical analysis, the instantaneous DGD is assumed to be a stationary process with Maxwellian distribution and mean value $\bar{\tau}$ [18]. The corresponding outage probability is

$$
\begin{aligned}
P_{\text {out }} & \triangleq \operatorname{Pr}\left\{\tau>\tau_{\max }\right\} \\
& =\operatorname{erfc}\left(\frac{2}{\sqrt{\pi}} \frac{\tau_{\max }}{\bar{\tau}}\right)+\frac{4}{\pi} \frac{\tau_{\max }}{\bar{\tau}} e^{-4 \tau_{\max }^{2} /\left(\pi \bar{\tau}^{2}\right)}
\end{aligned}
$$

In our approach, the idea is that, given a measure of the DGD at time $t_{0}$, we can derive a more accurate prediction of the statistical impact of PMD at time $t>t_{0}$ by exploiting some knowledge about the temporal dynamics of PMD. To this aim, we use the simple stochastic model in [12], in which the PMD vector $\vec{\tau}$ is modeled as an Ornstein-Uhlenbeck process in time, with drift coefficient $1 / T_{c}$ and diffusion coefficient $(\bar{\tau} / 2) \sqrt{\pi / T_{c}}$, where the PMD correlation time $T_{c}$ and the mean DGD $\bar{\tau}$ are two given parameters. The resulting conditional distribution of the PMD vector $\vec{\tau}(t)$ at time $t$ given the PMD vector $\vec{\tau}\left(t_{0}\right)$ at time $t_{0}$ is Gaussian. In particular, its components $\tau_{i}(t), i=1,2,3$, are three independent, jointly normal random variables, with mean values and variances

$$
\begin{aligned}
m_{i} & =\tau_{i}\left(t_{0}\right) e^{-\left(t-t_{0}\right) / T_{c}} \\
\sigma_{i}^{2} & =\sigma^{2}=\left(\pi \bar{\tau}^{2} / 8\right)\left[1-e^{-2\left(t-t_{0}\right) / T_{c}}\right]
\end{aligned}
$$

Therefore, the conditional distribution of the instantaneous DGD

$$
\tau(t)=\sqrt{\tau_{1}^{2}(t)+\tau_{2}^{2}(t)+\tau_{3}^{2}(t)}
$$

given $\tau\left(t_{0}\right)$ is a non-central chi distribution (also known as generalized Rice distribution) with three degrees of freedom [19]. As $t \rightarrow \infty$, the mean values in (2) vanish, the PMD vector approaches a stationary zero-mean Gaussian distribution with variance (per each component) $\pi \bar{\tau}^{2} / 8$, and the DGD approaches its well-known stationary Maxwellian distribution (the unconditional distribution) with mean DGD $\bar{\tau}$. We define the instantaneous conditional outage probability at time $t$ given the instantaneous DGD at time $t_{0}$

$$
P_{\text {out }}\left(t \mid \tau\left(t_{0}\right)\right) \triangleq \operatorname{Pr}\left\{\tau(t)>\tau_{\max } \mid \tau\left(t_{0}\right)\right\}
$$

Accounting for the non-central chi distribution of $\tau(t)$ given $\tau\left(t_{0}\right)$, the outage probability in (4) can be expressed as

$$
P_{\text {out }}\left(t \mid \tau\left(t_{0}\right)\right)=Q_{1.5}\left(\frac{\eta}{\sigma}, \frac{\tau_{\max }}{\sigma}\right)
$$

where $Q_{M}(x, y)$ is the generalized Marcum Q-function of order $M$, and

$$
\eta=\sqrt{\sum_{i=1}^{3} m_{i}^{2}}=\tau\left(t_{0}\right) e^{-\left(t-t_{0}\right) / T_{c}}
$$

is the non-centrality parameter of the chi distribution. By using the following result on the generalized Marcum Q-function of fractional order [20]

$$
\begin{aligned}
Q_{1.5}(x, y)=\frac{1}{2} \operatorname{erfc} & \left(\frac{y-x}{\sqrt{2}}\right)+\frac{1}{2} \operatorname{erfc}\left(\frac{y+x}{\sqrt{2}}\right) \\
& +\frac{1}{x \sqrt{2 \pi}}\left[e^{-(y-x)^{2} / 2}-e^{-(y+x)^{2} / 2}\right]
\end{aligned}
$$

and substituting (2) and (6) in (5), the conditional outage probability can be finally expressed as

$$
\begin{aligned}
P_{\text {out }}\left(t \mid \tau\left(t_{0}\right)\right)=\frac{1}{2} \operatorname{erfc}(\alpha) & +\frac{1}{2} \operatorname{erfc}(\beta) \\
& +\frac{1}{(\beta-\alpha) \sqrt{\pi}}\left(e^{-\alpha^{2}}-e^{-\beta^{2}}\right)
\end{aligned}
$$

where

$$
\beta, \alpha=\frac{2}{\sqrt{\pi}} \frac{\tau_{\max }}{\bar{\tau}}\left[\frac{1 \pm \tau\left(t_{0}\right) / \tau_{\max } e^{-t^{\prime}}}{\sqrt{1-e^{-2 t^{\prime}}}}\right]
$$

and $t^{\prime}=\left(t-t_{0}\right) / T_{c}$ is the normalized time. Fig. 1 shows the unconditional and instantaneous conditional outage probability as a function of the normalized time $t^{\prime}$, for different values of the initial DGD $\tau\left(t_{0}\right)$. The mean DGD is set to $\bar{\tau}=0.3 \tau_{\max }$. The initial DGD determines the transient values of the conditional 


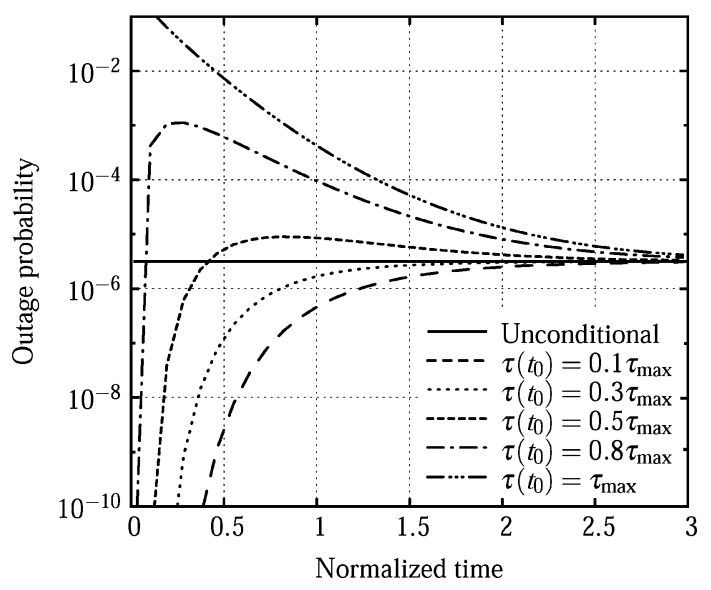

Fig. 1. Instantaneous conditional outage probability versus normalized time for a mean DGD $\bar{\tau}=0.3 \tau_{\max }$ and different values of the initial DGD $\tau\left(t_{0}\right)$. The unconditional outage probability is also shown as a reference.

outage probability. For $t^{\prime}=0$ the outage probability is either 0 or 1, depending on whether $\tau\left(t_{0}\right)$ is lower or higher than $\tau_{\max }$. On the other hand, for $t^{\prime} \rightarrow \infty$, the non-centrality parameter $\eta$ in (6) vanishes and, as expected, the conditional outage probability (8) converges to the unconditional one given by (1), regardless of the initial DGD. In particular, for small values of the initial DGD (e.g., $\tau\left(t_{0}\right) / \tau_{\max }=0.1,0.3$ ), (8) is always lower than (1) and converges to it from below, while for $\tau\left(t_{0}\right) \geq \tau_{\max }$, it is always higher and converges from above. Finally, for intermediate values of the initial DGD (e.g., $\tau\left(t_{0}\right) / \tau_{\max }=0.5,0.8$ ), the conditional outage probability given by (8) is lower than the unconditional one given by (1) during an initial period of time; then, it becomes higher than (1) and exhibits a maximum before converging to it. It is therefore clear that, for specific holding times, depending on the initial DGD, the conditional outage probability can be significantly lower than the unconditional one.

Since we are interested in setting up a lightpath during a finite time interval $\left[t_{1}, t_{2}\right]$, we define the average conditional outage probability in such an interval as

$$
\bar{P}_{\text {out }}\left(t_{1}, t_{2} \mid \tau\left(t_{0}\right)\right) \triangleq \frac{1}{t_{2}-t_{1}} \int_{t_{1}}^{t_{2}} P_{\text {out }}\left(t \mid \tau\left(t_{0}\right)\right) d t
$$

Since the variation time scale of $\alpha$ and $\beta$ in (8) is of the order of $T_{c}$, when $t_{2}-t_{1} \ll T_{c}$ the average outage probability (9) approaches the instantaneous one (8); on the other hand, when $t_{1}-t_{0} \gg T_{c}$ or $t_{2}-t_{0} \gg T_{c}$, the average outage probability (9) approaches the unconditional one (1). More in general, (9) is numerically evaluated by using (8) and resorting to some quadrature rule.

We conclude this Section with a few notes about the definition of outage probability and the dynamic PMD model adopted in this work. As regards the outage definitions in (1) and (4), a more accurate analysis should include other PMD parameters (e.g., the power-splitting ratio and second-order PMD) [21], [22]. However, as pointed out in [21], a tight upper bound for uncompensated systems can be obtained by integrating the joint distribution of the DGD $\tau$ and power splitting ratio $\rho$ in the rectangular region $\left\{\rho_{0} \leq \rho \leq 1-\rho_{0}, \tau \geq \tau_{\max }\right\}$, where $\tau_{\max }$ is the maximum instantaneous DGD value tolerated for $\rho=0.5$ (worst-case power splitting ratio), and $\rho_{0}\left(1-\rho_{0}\right)$ is the minimum (maximum) power-splitting ratio value for which outages may occur. Since no additional information about $\rho$ is gained by measuring the instantaneous DGD, the conditional distribution of $\rho$ is equal to the unconditional one, i.e., uniform in $\{0,1\}$ and independent of $\tau$. Therefore, the unconditional/conditional outage probability definition in (1)/(4), based on the first-order PMD approximation and worst-case power-splitting condition, coincide with the upper bound given in [21] for uncompensated systems, except for a multiplicative factor $1-2 \rho_{0}$, which can be practically neglected (slightly loosening the bound) or absorbed into the threshold outage probability value $P_{\mathrm{th}}$.

As regards the adopted dynamic PMD model, underlying the results in (5)-(9), its check against experimental data has shown a reasonable accuracy [12]. However, more complex and accurate models may be required to better represent PMD dynamics in installed networks [11], [23], [24]. They could be adopted in the provisioning scheme proposed in the next Section without significant changes, provided that computationally treatable formulas for the conditional outage probability can be derived and replaced in (8),(9).

\section{LIGHTPATH PROVISIONING BASED ON PMD CORRELATION}

A GMPLS-controlled transparent optical network is considered. RSVP-TE signaling protocol is extended to collect additive QoT parameters along a path, as in [4]: the inverse of OSNR to account for ASE, the square of the average DGD, the residual $\mathrm{CD}$, and the non linear phase shift $\phi_{\mathrm{NL}}$ to account for SPM. Each node has the knowledge of QoT parameters related to local (i.e., attached) links and ports. Each node can measure the DGD.

The proposed PMD-temporal-correlation (PTC) based lightpath provisioning scheme is described with a time chart in Fig. 2. Upon a lightpath request from source $s$ to destination $d$ with a holding time $\Delta t, s$ computes a path $p$ to $d$ ( $p$ is 1-2-3-4 in Fig. 2). Then, at time $t_{\mathrm{PT}}, s$ transmits an RSVP-TE Path message to $d$ to gather wavelength availability (in the standard Label Set object-LS) and QoT parameters $\left(\mathrm{OSNR}^{-1}, \mathrm{CD}, \phi_{\mathrm{NL}}\right.$ and $\left.\bar{\tau}^{2}\right)$. At time $t_{\mathrm{PR}}, d$ receives the Path message containing the list of wavelengths satisfying the wavelength continuity constraint, and the QoT parameters related to $p$. Then, the OSNR of the path is estimated [4]. For this purpose, the $\mathrm{CD}$ and $\phi_{\mathrm{NL}}$ parameters contained in the Path message are computed as penalty to the OSNR. Moreover, the non-modeled effects (e.g., cross-talk, polarization dependent loss) are accounted for with worst-case margins. Penalty and margins are subtracted from the received OSNR parameter. The computed $\mathrm{OSNR}_{f}$ (i.e., $\mathrm{OSNR}_{f}=\mathrm{OSNR}$-Penalty-Margins) is compared with a threshold OSNR $\min$.

If $\mathrm{OSNR}_{f}<\mathrm{OSNR}_{\min }$ (i.e., QoT blocking), or if no wavelength satisfies the wavelength continuity constraint (i.e., wavelength blocking), an RSVP-TE PathErr message is sent to $s$. Otherwise, besides the OSNR, the outage probability due to PMD has to be estimated. First, an available wavelength is selected (e.g., $\lambda_{5}$ ). Then, an RSVP-TE Resv message, carrying also $\bar{\tau}$ of $p$, is sent from $d$ to $s$ to reserve the selected wavelength and configure the optical cross-connects for probing. When, at 


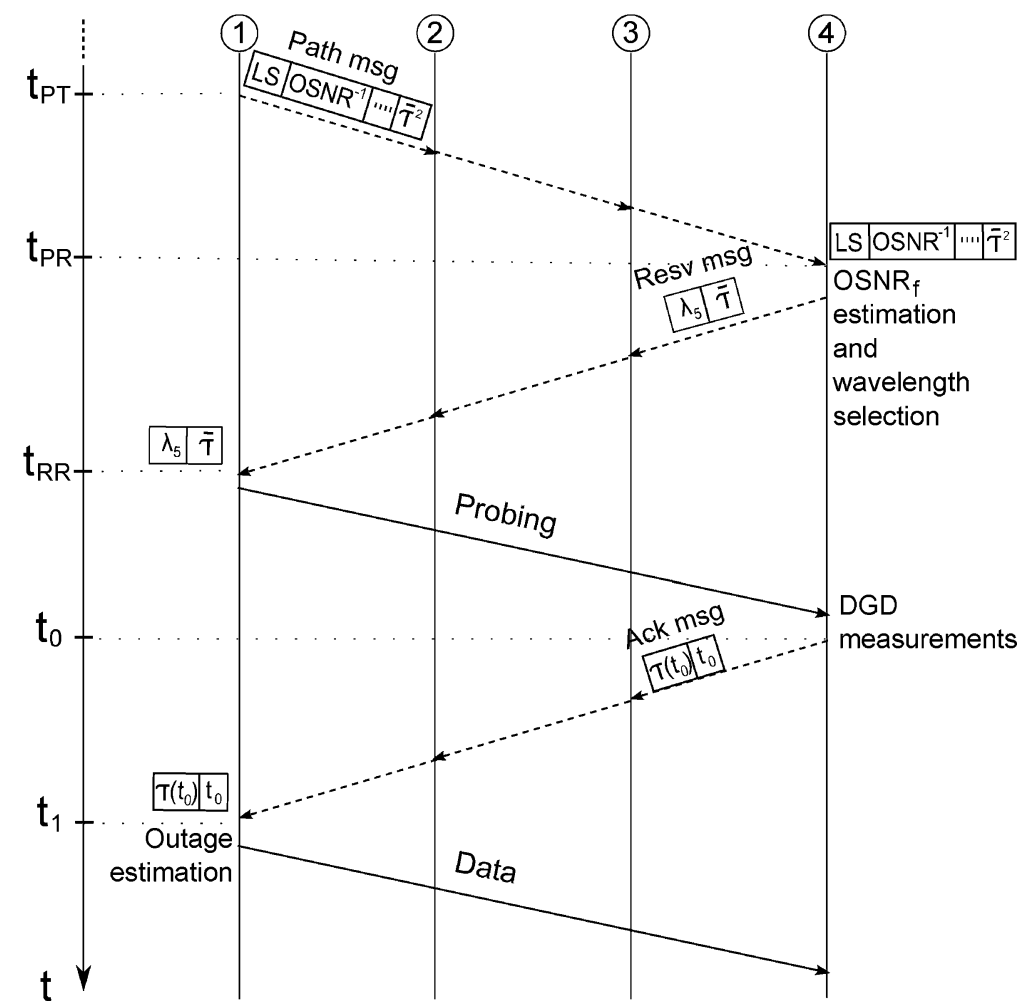

Fig. 2. Lightpath provisioning time chart.

time $t_{\mathrm{RR}}$, the Resv message reaches $s$, the lightpath is established for probing, and $s$ has the knowledge of $\bar{\tau}$ of $p$.

Then, the instantaneous DGD of $p$ is measured by sending probe traffic from $s$ along $p$ (in the data plane). At time $t_{0}, d$ measures the instantaneous DGD $\tau\left(t_{0}\right)$ and, along the control channels, it informs $s$ about: i) the measured DGD value $\tau\left(t_{0}\right)$ along $p$; ii) the time of measurement $t_{0}$. At time $t_{1}, s$ receives the DGD measurement information. If $\tau\left(t_{0}\right)>\tau_{\max }$ (i.e., QoT blocking) $s$ sends an RSVP-TE ResvErr message to $d$ to free resources along $p$. Otherwise, given $t_{2}=t_{1}+\Delta t$ (i.e., the lightpath tear down time), by exploiting $\bar{\tau}$ and $\tau\left(t_{0}\right), s$ computes $\bar{P}_{\text {out }}\left(t_{1}, t_{2} \mid \tau\left(t_{0}\right)\right)$ as in (9). If $\bar{P}_{\text {out }}\left(t_{1}, t_{2} \mid \tau\left(t_{0}\right)\right)>P_{\text {th }}$ (i.e., QoT blocking), a ResvErr is sent from $s$ to $d$ to free resources along $p$. Otherwise, the lightpath is finally activated and data traffic can be sent along $p$. If the lightpath set up fails due to QoT or wavelength blocking, $s$ performs further set up attempts along alternate paths.

\section{Performance Analysis}

In this section, the proposed PTC lightpath provisioning scheme is compared with the signaling approach with path feedback information (SAP) lightpath provisioning scheme [4]. SAP uses the model in (1), which does not consider PMD temporal correlation. With SAP, QoT blocking occurs if: i) $\operatorname{OSNR}_{f}<\mathrm{OSNR}_{\text {min }}$ as with PTC; ii) $P_{\text {out }}$, obtained from (1), is higher than $P_{\text {th }} . P_{\text {out }}$ is estimated at $d$ at time $t_{\mathrm{PR}}$, i.e., when the Path message is received. With SAP, if no wavelength or QoT blocking is experienced, data traffic starts to be transmitted at time $t_{\mathrm{RR}}$ without probing.

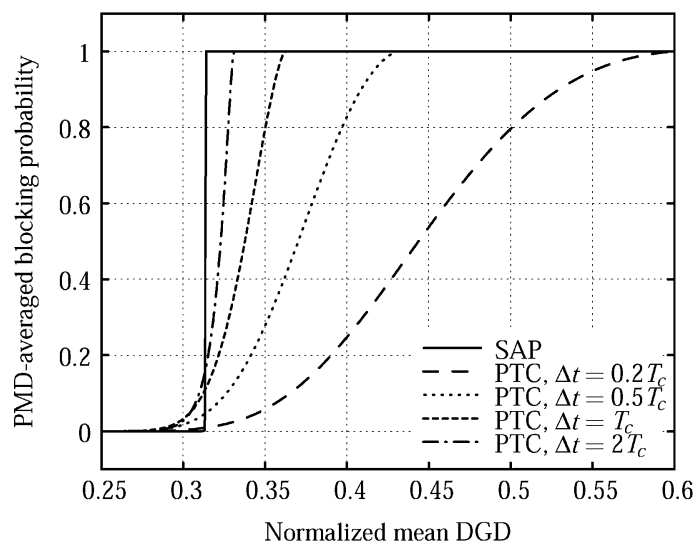

Fig. 3. QoT blocking probability due to PMD versus normalized mean DGD $\bar{\tau} / \tau_{\max }$ of the path with SAP and with PTC for different holding times $\Delta t$, averaged on $\tau\left(t_{0}\right)$.

\section{A. PMD Model Analysis}

To highlight the different behavior of the two considered lightpath provisioning schemes, Fig. 3 shows the PMD-averaged blocking probability (i.e., the QoT blocking probability due to PMD averaged on $\tau\left(t_{0}\right)$ ) for SAP and PTC as a function of the normalized mean DGD $\bar{\tau} / \tau_{\max }$ of the path. The considered threshold outage probability is $P_{\mathrm{th}}=10^{-5}$. For PTC, $t_{1}$ is set equal to $t_{0}$ and different holding times $\Delta t=t_{2}-t_{1}$ are considered.

With SAP, QoT depends only on $\bar{\tau}$. In this case, QoT blocking is a deterministic event that occurs when $P_{\text {out }}>P_{\text {th }}$, that is when $\bar{\tau}$ is higher than some threshold value, and the blocking 
TABLE I

DGD PARAMETERS AND Estimated OUTAGE PROBABILITY FOR TWO SAMPLE PATHS

\begin{tabular}{|c|c|c|c||c|c|c|c|}
\hline$p$ & $\bar{\tau}[\mathrm{ps}]$ & $\Delta t[\mathrm{~s}]$ & $\tau\left(t_{0}\right)[\mathrm{ps}]$ & $P_{\text {out }}$ & $\bar{P}_{\text {out }}\left(t_{1}, t_{2} \mid \tau\left(t_{0}\right)\right)$ & QoT with SAP & QoT with PTC \\
\hline$p_{1}$ & 13.1 & 654 & 22.6 & $2.8 \cdot 10^{-5}$ & $4.3 \cdot 10^{-5}$ & unacceptable & unacceptable \\
\hline$p_{1}$ & 13.1 & 577 & 3.2 & $2.8 \cdot 10^{-5}$ & $2.0 \cdot 10^{-8}$ & unacceptable & acceptable \\
\hline$p_{2}$ & 11.8 & 1200 & 24.8 & $1.8 \cdot 10^{-6}$ & $1.6 \cdot 10^{-5}$ & acceptable & unacceptable \\
\hline$p_{2}$ & 11.8 & 3376 & 17.2 & $1.8 \cdot 10^{-6}$ & $1.9 \cdot 10^{-6}$ & acceptable & acceptable \\
\hline
\end{tabular}

probability is either 0 or 1 depending on whether $\bar{\tau}$ is lower or higher than that threshold value.

With PTC, QoT depends also on the initial DGD $\tau\left(t_{0}\right)$ and holding time $\Delta t$; in this case, QoT blocking is a stochastic event that occurs when $\left.\bar{P}_{\text {out }}\left(t_{0}, t_{0}+\Delta t \mid \tau\left(t_{0}\right)\right)>P_{\text {th }}\right)$ and the PMD-averaged blocking probability-i.e., the probability that $\bar{P}_{\text {out }}\left(t_{0}, t_{0}+\Delta t\right)>P_{\text {th }}$, computed by averaging out the Maxwellian distribution of the initial DGD $\tau\left(t_{0}\right)$ - can assume intermediate values between 0 and 1 . Therefore, there is a finite probability that a path, that would be blocked by SAP, could be accepted by PTC, and a finite probability that a path, that would be accepted by SAP, could be blocked by PTC. However, Fig. 3 clearly shows that on average, for specific holding times, PTC permits to establish lightpaths along paths with a significantly larger mean DGD compared to SAP. As expected, for long holding times, PTC converges to SAP.

\section{B. Network Analysis}

The performance of the proposed PTC scheme is compared to the performance of SAP in a network scenario by means of a custom $\mathrm{C}++$ event-driven simulator. A Pan-European topology with 33 links and 17 nodes is considered [4]. Each link supports 40 wavelengths with $10-\mathrm{Gb} / \mathrm{s}$ NRZ modulation format. OSNR penalty due to CD and $\phi_{\mathrm{NL}}$ is modeled as in [4]. For each set up attempt, the measured DGD $\tau\left(t_{0}\right)$ is drawn from a Maxwellian distribution with mean value $\bar{\tau}=\left(\sum_{i \in p} D_{i}^{2} L_{i}\right)^{1 / 2}$, where $D_{i}=0.27 \mathrm{ps} / \sqrt{\mathrm{km}}$ is the PMD coefficient and $L_{i}$ the length of the $i$-th link in $p$. The correlation time is $T_{\mathrm{c}}=1000 \mathrm{~s}$, the maximum DGD $\tau_{\max }=40 \mathrm{ps}$, and the threshold outage probability is $P_{\text {th }}$. Lightpath requests follow a Poisson process and are uniformly distributed among all node pairs. Both lightpath inter-arrival and holding times are exponentially distributed with an average of $1 / \lambda$ and $1 / \mu$, respectively. Traffic load is expressed in Erlang as the ratio $\lambda / \mu$. $p$ is randomly selected among a set $P_{s, d}$, which contains all the paths within one hop from the shortest path.

PTC and SAP are compared in terms of lightpath blocking probability after $n$ set up attempts, defined as the ratio between the number of blocked lightpaths after up to $n$ set up attempts, and the number of lightpath requests. Lightpath set up attempts can fail due to QoT blocking or wavelength blocking. Up to $n=$ 3 set up attempts are performed along maximally link-disjoint paths.

Table I shows a set of cases in which two sample paths $p_{1}$ and $p_{2}$ (listed together with their DGD parameters) are either accepted or rejected due to PMD with SAP and PTC. Four lightpath holding time $\Delta t=t_{2}-t_{1}$ are considered and $P_{\mathrm{th}}$ is set to $10^{-5}$. Both paths do not experience QoT blocking due to low OSNR. The path $p_{1}$ has $\bar{\tau}=13.1 \mathrm{ps}$, which gives $P_{\text {out }}=$ $2.8 \cdot 10^{-5}$ higher than $P_{\text {th }}$. Therefore, $p_{1}$ is unacceptable with
SAP. Indeed, as shown in Fig. 3, paths with $\bar{\tau}>12.5$ ps (i.e., $\bar{\tau} / \tau_{\max }>0.31$ ), such as $p_{1}$, are always unacceptable with SAP. On the other hand, with PTC, $p_{1}$ can be either unacceptable (first line, $\Delta t=654 \mathrm{~s}$ and $\tau\left(t_{0}\right)=22.6 \mathrm{ps}$ ) or acceptable (second line, $\Delta t=577 \mathrm{~s}$ and $\left.\tau\left(t_{0}\right)=3.2 \mathrm{ps}\right)$.

Regarding the path $p_{2}$, it has $\bar{\tau}<12.5 \mathrm{ps}$, thus it is always acceptable with SAP. Again, with PTC, $p_{2}$ can be either unacceptable (third line, $\Delta t=1200 \mathrm{~s}$ and $\tau\left(t_{0}\right)=24.8 \mathrm{ps}$ ) or acceptable (fourth line, $\Delta t=3376 \mathrm{~s}$ and $\tau\left(t_{0}\right)=17.2 \mathrm{ps}$ ). In particular, the third line of the table shows a case where PTC, exploiting $\tau\left(t_{0}\right)$ as additional information with respect to SAP, refuses the lightpath along $p_{2}$ because of the excessive $\tau\left(t_{0}\right)$.

Fig. 4 shows the lightpath blocking probability versus the network load, obtained by varying $1 / \lambda$ when $1 / \mu=500$ s, i.e., with a fixed average holding time. $P_{\text {th }}$ is set to $10^{-6}$ in Fig. 4(a), and to $10^{-5}$ in Fig. 4(b).

Lightpath blocking probability decreases while $n$ increases, as more feasible paths are tested. Fig. 4(a) shows that PTC significantly reduces the blocking probability with respect to SAP at each $n$-th set up attempt. At loads lower than 400 Erlang, SAP achieves a constant and high blocking probability. For these loads QoT blocking is the dominant contribution, which is not influenced by lightpath mean inter-arrival and holding times with the uncorrelated PMD model. In particular, the uncorrelated PMD model causes the rejection of all paths longer than $1586 \mathrm{~km}$ (as reported by Table II).

On the contrary, PTC allows to accept lightpaths with a length up to $2104 \mathrm{~km}$ (as reported by Table II). PTC experiences an almost constant blocking probability at loads lower than $400 \mathrm{Er}$ lang, since with the PMD correlation based model QoT blocking is influenced only by the holding time and not by the inter-arrival time, as shown in (9). For loads higher than 400 Erlang, the blocking probability of both schemes further increases since the wavelength blocking becomes dominant.

By comparing Figs. 4(a) and (b), it can be noticed that the blocking probability with both SAP and PTC when $P_{\text {th }}=10^{-5}$ is lower than the one experienced when $P_{\text {th }}=10^{-6}$. Fig. 4(b) shows that PTC is able to significantly reduce the blocking probability at the first set up attempt. Differently from Fig. 4(a), for medium loads SAP experiences a null blocking when $n=2$, since paths with acceptable QoT are discovered in successive set up attempts. This is due to the less stringent limit on the outage probability, i.e., $P_{\mathrm{th}}=10^{-5}$, compared to $P_{\mathrm{th}}=10^{-6}$ of Fig. 4(a). Fig. 4(b) confirms that both models are not influenced by the average inter-arrival time. For medium loads, PTC experiences a non-null blocking probability for $n=2$. This is explained with Fig. 3, which shows a region in which the PMD-averaged blocking probability predicted with PTC (by exploiting the knowledge of $\left.\tau\left(t_{0}\right)\right)$ is actually higher than that predicted with SAP (which does not exploit $\tau\left(t_{0}\right)$ ). For $n=3$ 


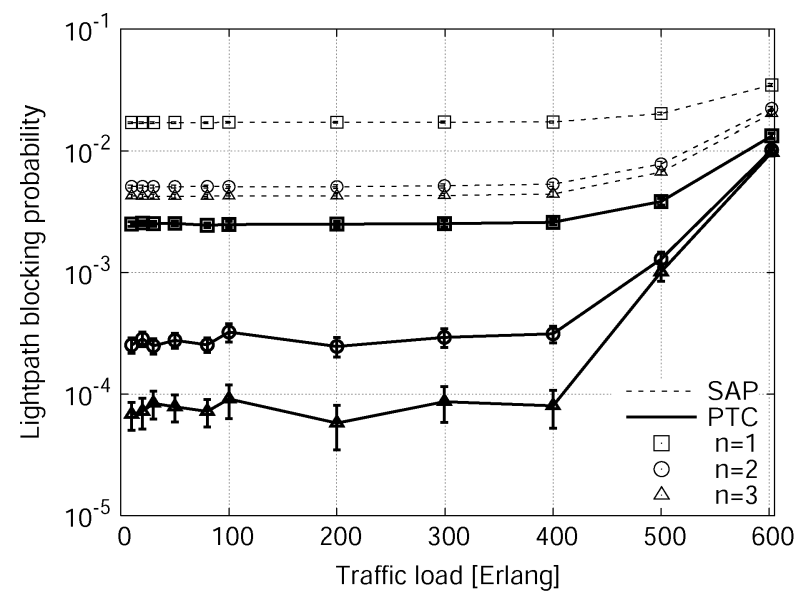

(a)

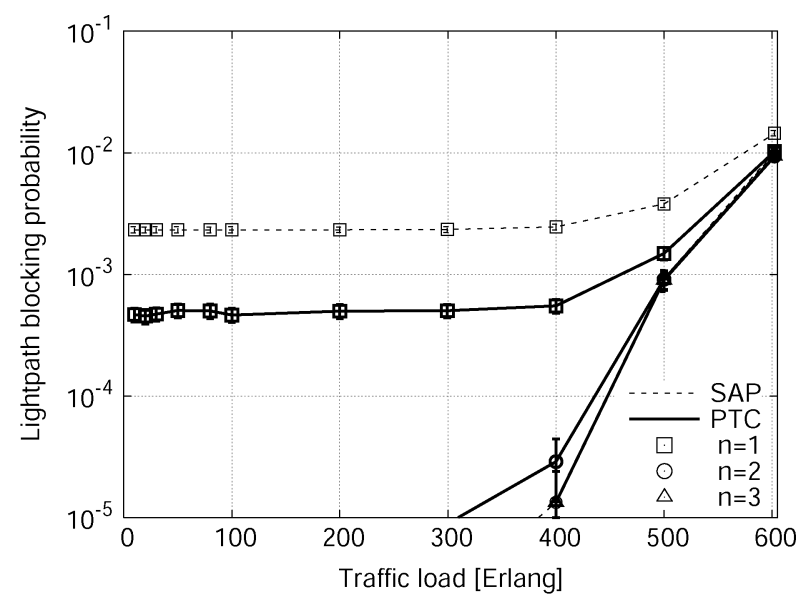

(b)

Fig. 4. Lightpath blocking probability versus traffic load [Erlang] at varying $1 / \lambda$ with $1 / \mu=500 \mathrm{~s}$. (a) With $P_{\mathrm{th}}=10^{-6}$, (b) with $P_{t h}=10^{-5}$.

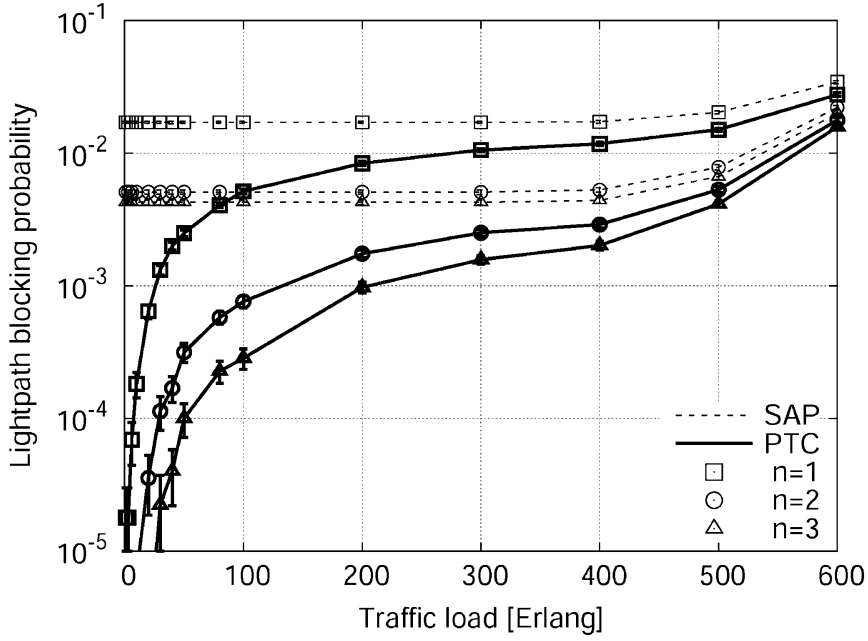

(a)

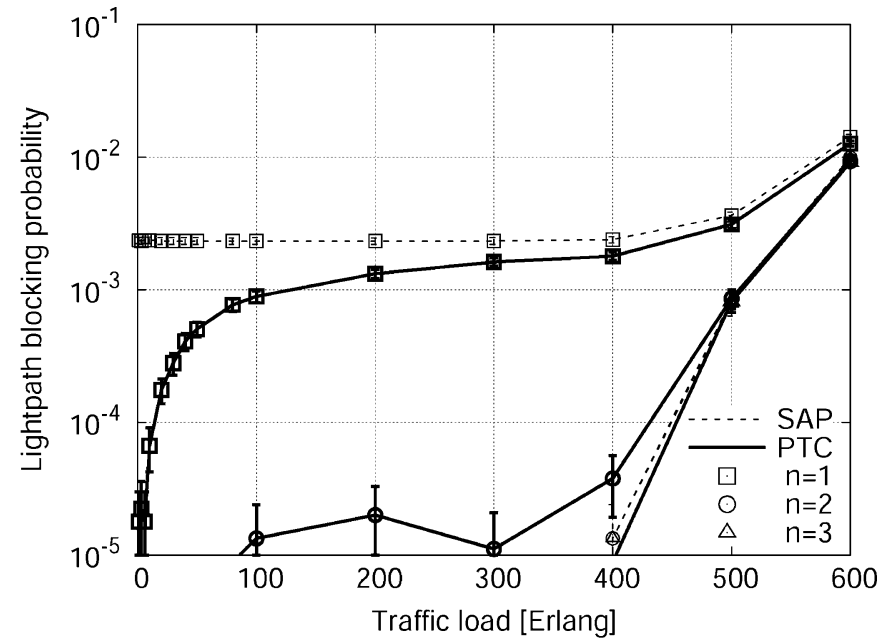

(b)

Fig. 5. Lightpath blocking probability versus traffic load [Erlang] at varying $1 / \mu$ with $1 / \lambda=10$ s. (a) With $P_{\mathrm{th}}=10^{-6}$, (b) with $P_{\mathrm{th}}=10^{-5}$.

TABLE II

MAXIMUM LENGTH OF AN ACCEPTED LIGHTPATH [KM]

\begin{tabular}{|c||c|c|}
\hline$P_{t h}$ & SAP & PTC \\
\hline $10^{-6}$ & 1586 & 2104 \\
\hline $10^{-5}$ & 1927 & 2267 \\
\hline
\end{tabular}

both SAP and PTC experience a null QoT blocking. Finally, with $P_{\text {th }}=10^{-5}$, paths with length up to 1927 and $2267 \mathrm{Km}$ are acceptable with SAP and PTC, respectively (as reported by Table II).

Fig. 5 shows the blocking probability versus the network load, obtained by varying $1 / \mu$ when $1 / \lambda=10 \mathrm{~s}$, i.e., with a fixed average inter-arrival time. $P_{\text {th }}$ is set to $10^{-6}$ in Fig. 5(a), and to $10^{-5}$ in Fig. 5(b).

Fig. 5(a) confirms that when the limit on the outage probability is stringent, PTC significantly decreases the blocking probability with respect to SAP at each $n$-th set up attempt. In particular, for low loads (corresponding to short holding times) QoT blocking approaches zero even with stringent $P_{\text {th }}=10^{-6}$. Then, for increasing load, PTC blocking probability increases and approaches SAP blocking, since the lightpath holding time is more likely to be higher than $T_{c}$, where both models give similar outage probability estimates. Again, for loads higher than 400 Erlang, the blocking probability of both schemes increases since the wavelength blocking becomes dominant. Similarly to Fig. 4(b) obtained with the same $P_{\text {th }}$, in Fig. 5(b) PTC overcomes SAP at the first attempt, while SAP achieves a null QoT blocking at the second attempt.

Table II refers to Figs. 4 and 5, and summarizes the maximum length values of the accepted lightpaths (here named as network transparency size), for both SAP and PTC. The table shows that PTC permits to increase the network transparency size, for both $P_{\text {th }}$ values. In particular, PTC permits to increase the transparency domain size for the considered scenario with respect to SAP by $30 \%$ and $18 \%$ when $P_{\text {th }}$ is $10^{-6}$ and $10^{-5}$, respectively.

\section{CONCLUSION}

In this paper, a lightpath provisioning scheme, called PMD-temporal-correlation based (PTC) lightpath provisioning 
scheme, has been proposed for GMPLS-controlled dynamic transparent optical networks. PTC exploits a PMD prediction model, based on the fact that DGD evolution is correlated in time. Therefore, if a lightpath does not experience a channel outage at a specific time, it is supposed that the outage is not experienced for a subsequent time range.

PTC exploits RSVP-TE extensions to estimate physical impairment parameters during lightpath set up. Additionally, PTC enforces a monitoring phase in which DGD is measured in order to estimate the outage probability within the required lightpath holding time.

Simulation results show the benefits of the proposed PTC lightpath provisioning scheme with respect to a classical QoT-aware lightpath provisioning scheme. In particular, PTC strongly reduces the lightpath blocking probability with respect to the other scheme. Moreover, PTC allows an increase in the transparency domain size, since the PMD temporal correlation model permits establish of lightpaths along paths that would be rejected by the classical model.

\section{REFERENCES}

[1] B. Ramamurthy, D. Datta, H. Feng, J. P. Heritage, and B. Mukherjee, "Impact of transmission impairments on the teletraffic performance of wavelength-routed optical networks," J. Lightw. Technol., vol. 17, no. 10, pp. 1713-1723, Oct. 1999.

[2] J. Strand, A. L. Chiu, and R. Tkach, "Issues for routing in the optical layer," IEEE Commun. Mag., vol. 39, no. 2, pp. 81-87, Feb. 2001.

[3] D. Penninckx and C. Perret, "New physical analysis of 10-Gb/s transparent optical networks," IEEE Photon. Technol. Lett., vol. 15, no. 5, pp. 778-780, May 2003.

[4] F. Cugini, N. Sambo, N. Andriolli, A. Giorgetti, L. Valcarenghi, P. Castoldi, E. Le Rouzic, and J. Poirrier, "Enhancing GMPLS signaling protocol for encompassing quality of transmission (QoT) in all-optical networks," J. Lightw. Technol., vol. 26, no. 19, pp. 3318-3328, Oct. 2008.

[5] A. Morea, N. Brogard, F. Leplingard, J. Antona, T. Zami, B. Lavigne, and D. Bayart, "QoT function and A* routing: An optimized combination for connection search in translucent networks," OSA J. Opt. Netw., vol. 7, no. 1, pp. 42-61, 2008.

[6] Y. Pointurier, M. Brandt-Pearce, and S. Subramaniam, "Analysis of blocking probability in noise- and cross-talk-impaired all-optical networks," IEEE/OSA J. Opt. Commun. Netwo. (JOCN), vol. 1, no. 6, pp. 543-554, Nov. 2009.

[7] D. C. Kilper, R. Bach, D. J. Blumenthal, D. Einstein, T. Landolsi, L. Ostar, M. Preiss, and A. E. Willner, "Optical performance monitoring," J. Lightw. Technol., vol. 22, no. 1, pp. 294-304, Jan. 2004.

[8] N. Sambo, C. Pinart, E. Le Rouzic, F. Cugini, L. Valcarenghi, and P. Castoldi, "Signaling and multi-layer probe-based schemes for guaranteeing QoT in GMPLS transparent networks," in Proc. OFC 2009, San Diego, CA, Feb. 2009.
[9] G. Martinelli and A. Zanardi, "GMPLS signaling extensions for optical impairment aware lightpath setup," in IETF Draft-Martinelli-Ccamp-Optical-Imp-Signaling-02.Txt, Jul. 2009 [Online]. Available: http://tools.ietf.org/id/draft-martinelli-ccamp-optical-imp-signaling-02.html

[10] G. Bernstein, Y. Lee, and W. Imajuku, "Framework for GMPLS and PCE control of wavelength switched optical networks," in Draft-Bernstein-Ccamp-Wavelength-Switched-03.Txt, Oct. 2007 [Online]. Available: http://tools.ietf.org/html/draft-bernstein-ccamp-wavelength-switched-03

[11] M. Brodsky, M. Boroditsky, P. Magill, N. Frigo, and M. Tur, "A hinge model for the temporal dynamics of polarization mode dispersion," in Proc. 17th Ann. Meeting IEEE Lasers Electro-Optics Society LEOS, Nov. 2004, pp. 90-91.

[12] C. Antonelli, A. Mecozzi, M. Brodsky, and M. Boroditsky, "A simple analytical model for PMD temporal evolution," in Proc. OFC 2006, Mar. 2006, Paper OWJ4.

[13] O. Karlsson, J. Brentel, and P. Andrekson, "Long-term measurement of PMD and polarization drift in installed fibers," J. Lightw. Technol., vol. 18, no. 7, pp. 941-951, Jul. 2000.

[14] C. Allen, P. Kondamuri, D. Richards, and D. Hague, "Measured temporal and spectral PMD characteristics and their implications for network-level mitigation approaches," J. Lightw. Technol., vol. 21, no. 1, pp. 79-86, Jan. 2003.

[15] M. Brodsky, N. Frigo, M. Boroditsky, and M. Tur, "Polarization mode dispersion of installed fibers," J. Lightw. Technol., vol. 24, no. 12, pp. 4584-4599, Dec. 2006.

[16] J. Jiang, S. Sundhararajan, D. Richards, S. Oliva, M. Sullivan, and R. Hui, "PMD monitoring in traffic-carrying optical systems," in Proc. ECOC 2008, Sep. 2008.

[17] M. N. Petersen, N. Sambo, N. Andriolli, and M. Scaffardi, "PMD monitoring using optical sideband filtering," in Proc. LEOS 2009, Oct. 2009, pp. 129-130.

[18] G. J. Foschini and C. D. Poole, "Statistical theory of polarization dispersion in single mode fibers," J. Lightw. Technol., vol. 9, no. 11, pp. 1439-1456, 1991.

[19] J. G. Proakis, Digital Communications, 4th ed. New York: McGrawHill, 2001.

[20] A. Annamalai, C. Tellambura, and J. Matyjas, "A new twist on the generalized marcum Q-function $\mathrm{QM}(\mathrm{a}, \mathrm{b})$ with fractional-order $\mathrm{M}$ and its applications," in Proc. 6th IEEE CCNC, Jan. 2009, pp. 1-5.

[21] E. Forestieri and G. Prati, "Exact analytical evaluation of second-order PMD impact on the outage probability for a compensated system," $J$. Lightw. Technol., vol. 22, no. 4, pp. 988-996, Apr. 2004.

[22] P. Winzer, H. Kogelnik, and K. Ramanan, "Precise outage specifications for first-order PMD," IEEE Photon. Technol. Lett., vol. 16, no. 2, pp. 449-451, Feb. 2004.

[23] C. Antonelli, C. Colamarino, A. Mecozzi, and M. Brodsky, "A model for temporal evolution of PMD," IEEE Photon. Technol. Lett., vol. 20, no. 12, pp. 1012-1014, Jun. 2008.

[24] D. Yevick, M. Reimer, and M. O'Sullivan, "Simplified transition matrix analysis of the hinge model," J. Opt. Soc. Amer. A vol. 26, no. 3, pp. 710-714, 2009.

Author biographies not included at authors' request due to space constraints. 